\title{
A study on variation in position of an Indian station due to solid earth tides
}

\author{
Jayanta Kumar Ghosh* and Shray Pathak \\ Department of Civil Engineering, Indian Institute of Technology, Roorkee 247 667, India. \\ *Corresponding author. e-mail: gjkumfce@yahoo.com
}

In many geodetic analyses, it is important to consider the effect of earth tide on the instantaneous position of a station and its subsequent influence on the computation and interpretation of time series of coordinates as well as related data products. The tidal effect and temporal variations in the position of the IGS (International Global Navigational Satellite Systems [GNSS] Service) stations at Hyderabad (India), Ankara (Turkey) and Beijing Fangshan (China), due to solid earth tides has been studied. Surface tidal displacement of the station has been computed on daily basis for a month, based on the concept of gravity. Further, mean daily coordinates of the station been computed using static precise point positioning (PPP) method for a month. Results show that the station undergoes temporal displacements and its coordinates vary continuously within a day and all the days in the month. The maximum range in vertical displacement of the station has been found to be about $48 \mathrm{~cm}$ in a day over a period of a month and that along the north and east directions is respectively $8 \mathrm{~cm}$ and $14 \mathrm{~cm}$. This is the maximum range but the mean value in the vertical displacement is $6 \mathrm{~cm}$ and along north and east is $1.7 \mathrm{~cm}$ and $0.09 \mathrm{~cm}$, respectively. The ranges in variation in the mean value of geodetic latitude, longitude, and height of the station have been found to be $1.23,2.73$, and $3.52 \mathrm{~cm}$, respectively. Further, it has been found that the tidal oscillations follow some periodicity, and thus need to be studied independently for all stations.

\section{Introduction}

Earth tide is a temporal deformation in the earth's shape and size due to the presence of the heavenly bodies around the Earth. Deformation is temporal in nature and it rebounds back after the removal of the effect. The earth tide occurs as a result of gravitational forces between the Earth, Moon, Sun, and the centrifugal forces of the rotation system. Therefore the tidal forces depend upon the location of the point on the Earth and mutual position of the Moon and the Sun with respect to that point. The Sun exerts high gravitational force with respect to Moon, but due to proximity of the Moon, Moon contributes around $60-70 \%$ of the tidal force. The temporal deformations of the solid earth can be calculated by knowing its elastic behaviour. Love numbers are introduced to define the elasticity of Earth (Love 1944), further described in Munk and MacDonald (1975). This technique expresses the radial and transverse displacement of a point on the Earth's crust in terms of Love and Shida numbers ( $\mathrm{h}$ and $\mathrm{l}$ respectively), in addition to the perturbation in the geopotential field using the Love number k (Mathews et al. 1997).

Great efforts have been made to accurately measure the earth tides (Richter and Warburton 1998). There have been many theoretical studies of the Earth's structure and tidal response after the study done by Love in 1909. Tidal deformations were studied for a spherically symmetric, perfectly elastic, and isotropic Earth (Longman 1963; Saito

Keywords. Solid earth tides; positional variation; surface displacements. 
1967; Farrell 1972). The response of the Earth to luni-solar attraction is expressed by amplitudes and phases of tidal constituents, together with the ocean tide loading (OTL) effects (Lambert et al. 1998).

The tidal response is mainly related to the Earth's elastic properties and local variations in elastic structure (Mantovani et al. 2005; Fu and Sun 2007). Thus, the Earth's tidal response can be used to investigate the inner structure. Recent studies have shown theoretically and through the use of simulated data how unmodelled periodic signals (such as ocean tide loading and errors in solid earth tide models) propagate into GPS coordinate time series at different frequencies (Penna and Stewart 2003; Stewart et al. 2005; Penna et al. 2015). It is well known that the position of a point on the Earth's surface varies over a range of temporal scales due to the elastic response of the crust to the external tide generating potential (TGP) (Melchoir 1983). The resultant response is called the solid earth tide (also termed the earth body tide), and can account for displacements up to $\sim 0.4 \mathrm{~m}$ at predominantly semi-diurnal and diurnal frequencies (Lambeck 1988).

On the global scale, GPS network observations have been conducted by Schenewerk et al. (2001), who estimated the vertical tidal displacements of 353 globally distributed GPS stations for eight major semi-diurnal and diurnal constituents using 3 years of observations and found large-scale systematic observation vs. model differences.

The study done by Yuan et al. (2009) used GPS data from a dense, continuous (albeit local) network in Hong Kong to evaluate the internal precision of GPS tidal displacement estimates. The results then showed that the misfits for the major semi-diurnal and diurnal constituents (except K1 and $\mathrm{K} 2$ ) are $<0.5$ and $1.0 \mathrm{~mm}$, respectively, for the horizontal and vertical components, implying that the GPS measurement error may not be a limiting factor to scrutinize body tide and OTL models. The study done by Yuan and Chao (2012) succeeded in demonstrating the precision of GPS tidal displacement estimates down to the level of $\sim 0.1$ $\mathrm{mm}$ (horizontal) and $\sim 0.3 \mathrm{~mm}$ (vertical). Tidal oscillation has a strictly definite periodicity, therefore, there is need to study solid earth tides so that the corrections can be applied on both the GPS data and ' $\mathrm{g}$ ' value to calculate it for a particular place and time. In any geodetic analysis, it is important to consider how this process may affect the instantaneous site position, and hence influence the computation and interpretation of coordinate time series and related data products.

Further, Haritonova Diana (2012) uses the special program to describe the vertical tide displacements in the territory of Latvia. Tidal deformations are the earth crust vertical movements with maximum amplitude of up to $30 \mathrm{~cm}$.

However, there is a need to calculate the tidal deformations and their effect on India. The study is to calculate the earth tide variation of the International GNSS Service (IGS) Station at Hyderabad (India), Ankara (Turkey), and Beijing Fangshan (China). The daily and monthly variations are calculated for the month of November, 2013 at IGS stations.

\section{Theory}

The tidal force arises because of the gravitational attraction of the heavenly bodies around the Earth which causes tidal acceleration. The tidal acceleration at any point on the surface of the Earth is the difference between the acceleration caused by the attraction of the external body and the orbital acceleration, i.e., acceleration which the Earth undergoes as whole, explained by Agnew (2007). Theoretically earth tide phenomenon is explained by tidal phenomenon by applying Newton's laws of motion. The gravitational potential is inversely proportional to the square of the distance between the body and the tide generating body whereas the tidal potential is inversely proportional to the cube of the distance between them. The tidal force is expressed by the more preferred scalar quantity, i.e., tidal potential. Tidal potential is calculated in two ways: either with the use of angular distance or geographical coordinates to define the position on the Earth. The expression for the tidal potential is described by Munk and Cartwright (1966) and the position is defined by the angular distance. The distance between the two masses (Earth and tide generating body) and the angular distance between the center of the tide generating body and the point on the surface of Earth is taken as the function of time. Thus it implements that the tidal potential is also a function of time.

If the tide generating body is Moon, and the point is on the surface of Earth where the tidal potential is to be calculated then, $R / D=1 / 60$, where $R$ is the radius of Earth and $D$ is the distance between the two masses. In the case of Sun, $R / D=1 / 23,000$.

Therefore for second degree, the magnitude of $\mathrm{V}_{\text {tid }}$ is proportional to GM/D ${ }^{3}$. By normalizing this quantity to make value for the Moon equal to 1 , then the value for Sun is 0.46 , for Venus is $5 \times 10^{-5}$ and for Jupiter is $6 \times 10^{-6}$. Hence, the Moon and Sun contribute effectively and can be visible in the actual measurements.

In the other method, the distance is defined by geographical coordinates (Agnew 2007). In this the tidal potential depends upon the latitude and longitude of the tide generating body as well as 
the point where the tidal potential is to be calculated. The tidal potential when divided by the acceleration due to gravity gives the units of potential. From this the tidal potential can be computed directly. The location of the Moon and the Sun from the celestial coordinates is converted into the geographical coordinates and the distance is converted by using the standard transformations (McCarthy and Petit 2004, Chapter 4).

The Love and Shida numbers are the dimensionless parameters used to define the elasticity of the Earth. For the real Earth, the effective Love numbers are dependent on the site latitude and tidal frequency (Wahr 1981; Mathews et al. 1997; Dehant et al. 1999). For attaining better accuracy of less than $1 \mathrm{~mm}$ in modelling of site displacements, various effects have to be considered on the Love numbers. The motions of the Moon and the Sun as observed in the geocentric coordinate system are predictable by harmonic functions of the periodic variations of their respective elliptical orbits with high accuracy. Instead of two Love numbers $\mathrm{h}$ and $\mathrm{l}$ used for a spherical Earth (Mathews et al. 1997), the seven Love numbers $\left(\mathrm{h}^{(0)}, \mathrm{h}^{(2)}\right.$, $\mathrm{h}^{\prime}$ ) and $\left(\mathrm{l}^{(0)}, \mathrm{l}^{(1)}, \mathrm{l}^{(2)}, \mathrm{l}^{\prime}\right)$ are used to describe the surface tidal displacement of an ellipsoidal Earth. The surface displacement $\vec{u}$ because of the tidal term of frequency $f$ is expressed in terms of frequency and latitude-dependent Love numbers (Mathews et al. 1997; Dehant et al. 1999). The components of the tidal displacements of degree-2 can be explained as:

For a diurnal cycle of frequency $f$ :

$$
\begin{aligned}
& \vec{u}=-\sqrt{\frac{5}{24 \pi}} H_{f}\left\{3 h(\theta) \sin \theta \cos \theta \sin \left(\varphi_{f}+\phi\right) \hat{e}_{r}\right. \\
& +\left[3 l(\theta) \cos 2 \theta-3 l^{(1)} \sin ^{2} \theta+\sqrt{\frac{24 \pi}{5}} l^{\prime}\right] \sin \left(\varphi_{f}+\phi\right) \hat{e}_{\theta} \\
& +\left[\left(3 l(\theta)-\sqrt{\frac{24 \pi}{5}} l^{\prime}\right) \sin \theta-3 l^{(1)} \sin \theta \cos 2 \theta\right] \\
& \left.\times \cos \left(\varphi_{f}+\phi\right) \hat{e}_{\phi}\right\}
\end{aligned}
$$

where $H_{f}$ is the amplitude $(\mathrm{m})$ of the tidal term of frequency $f, \theta$ is the geocentric latitude of station, $\Phi$ is the east longitude of station, $\varphi_{f}$ is the tide argument for tidal constituent with frequency $f, \hat{e}_{r}$ is the unit vector in radial direction, $\hat{e}_{\phi}$ is the unit vector in the east direction, and $\hat{e}_{\theta}$ is the unit vector at right angles to $\hat{e}_{r}$ in the northward direction.

\subsection{Earth tide variation}

As the earth tide is, because of the Moon and the Sun, depends upon their position and the distance from the point where the effect of the tide is to be calculated. To calculate the earth tides we require the latitude and longitude of both the tide generating body and the point where the earth tide is to be calculated. If we observe the earth tide at a particular point/position, we can say that for a diurnal cycle it can be expected that each point faces two high and low tides. High tide is that when the point on the Earth experiences maximum tidal force and low tide is that when it experiences minimum tidal force. Though the high and low tides occur twice in a day and are different in magnitudes, it is expected to occur in approximately 12 hours. Therefore in approximately 6 hours, each point faces high and low tides.

With the variation in the position of the point on the Earth and the tide generating bodies, the Earth experiences two types of tides: Spring tide and Neap tide. Spring tide is that in which the Sun, Earth, and Moon all are aligned in the same line, due to which they form constructive interface and the Earth experiences the maximum tidal force. In spring tide, the phase of the Moon is either new moon or full moon. Neap tide is that when the Sun, Earth, and Moon are aligned in perpendicular line, due to which they form destructive interface and the Earth experiences the minimum tidal force. In neap tide, the phase of the Moon is either in first quarter or third quarter. While ocean tides can be easily measured relative to the solid Earth, solid earth tides are easily measured only with satellite systems or sensitive gravimeters. Also, the solid earth tide is a very smooth function around the Earth. For this reason, differential positioning systems, such as differential carrier phase GPS, can frequently ignore solid earth tide effects. Global geodetic networks and GPS carrier phase precise point positioning must include the effect of solid earth tide.

\section{Methodology}

The solid earth tide is calculated at the IGS station, Hyderabad (India), Ankara (Turkey) and Beijing Fangshan (China) for the month of November 2013. For observing the variation at a station for a particular day, the gravity method is used. This method is implemented through the program 'Solid'. The displacements in all the three directions, that is along the north, east and vertical directions are calculated on a daily basis at a 1-min interval. The program 'Solid' is offered by Dennis Milbert (2011). This code is an implementation of the solid earth tide computation found in section 7.1.2 of the IERS Conventions (2003), IERS Technical Note No. 32. The program asks for the date and geodetic location and gives the tidal amplitude 
as an output of that location. It gives the amount of displacements in all the three directions with reference to the input values of the geodetic location.

\section{Observations}

\subsection{By gravity method}

\subsubsection{For IGS Hyderabad station}

The program 'Solid' is the implementation of the gravity method. The program asks for the date and geodetic latitude and longitude. This software gives the solid earth tide components in all the three directions, i.e., north, east, and vertical. The time is given in GPS time and the values are given at an interval of $1 \mathrm{~min}$. The program gives the amount of displacements occuring in all directions with respect to the input geodetic coordinates. It gives 1440 values of displacements in all the three directions at $1 \mathrm{~min}$ intervals for one day. The value of displacements for the whole month along the vertical direction is shown in figure 1 . The y-axis shows the amount of displacement occuring along the height of the position in meters. The figure shows the variation in the displacement in the height of the station for the whole month. The maximum range of a day for the whole month comes to $48 \mathrm{~cm}$. The figure also shows that the two high and low tides occur in a single particular day, though they are not of same magnitude. This is because of the diurnal cycle of the Earth that a point faces two high and low tides for a particular day but not of same magnitude. The maximum vertical displacement is about $30 \mathrm{~cm}$ and minimum is about -18 $\mathrm{cm}$ for that particular day.

Figure 2 shows the displacement of the station along the north direction with respect to the input value of the latitude entered into the program. The expansion force along the north side is less as compared to the compressive force. The range of the displacement is about $8 \mathrm{~cm}$ with maximum displacement along north side is $1 \mathrm{~cm}$ and minimum displacement is $-7 \mathrm{~cm}$.

Figure 3 shows the displacement of the station along the east direction with respect to the input value of the longitude entered in the program. The figure shows the variation in the displacements along the east direction. The range of the displacements is about $14 \mathrm{~cm}$, the maximum displacement along the east side is about $7 \mathrm{~cm}$ and minimum displacement is $-7 \mathrm{~cm}$.

Figures 1,2, and 3 clearly show the displacements of the position in all the three directions for the month of November. This shows that due to the solid earth tides, the displacement occurs in all the three directions at $1 \mathrm{~min}$ intervals. To compare the same, tidal displacements are computed for other IGS stations, i.e., Ankara (Turkey), and Beijing Fangshan (China).

\subsubsection{For IGS Ankara station}

The displacements are computed along all the three directions per minute for a month. The maximum displacement along the upward vertical direction comes to $26 \mathrm{~cm}$ and maximum downward displacement is $17 \mathrm{~cm}$. Thus the maximum range of displacement per day for a month comes to $43 \mathrm{~cm}$. The value of displacements for the whole month along the vertical direction is shown in figure 4 . The yaxis shows the amount of displacement occuring along the height of the position in meters. The figure shows the variation in the displacement in the height of the station for the whole month.

Similarly, the displacements along the other directions are also computed and the ranges in other directions are observed. The maximum positive displacement along the north direction comes to $0.7 \mathrm{~cm}$ and negative displacement is $7 \mathrm{~cm}$, thus the maximum range of a day for a month comes to $7.7 \mathrm{~cm}$ (figure 5).

The maximum positive displacement along the east direction comes to $7 \mathrm{~cm}$ and negative displacement is also $7 \mathrm{~cm}$. Thus the maximum range of the day for the month is $14 \mathrm{~cm}$ (figure 6).

\subsubsection{For IGS Beijing Fangshan station}

The displacements are computed along all the three directions per minute for a month. The maximum positive displacement along the vertical direction comes to $25 \mathrm{~cm}$ and maximum negative displacement is $17 \mathrm{~cm}$. Thus the maximum range of displacement per day for a month comes to $42 \mathrm{~cm}$. The value of displacements for the whole month along the vertical direction is shown in figure 7 . The $y$-axis shows the amount of displacement occuring along the height of the position in meters. The figure shows the variation in the displacement in the height of the station for the whole month.

Similarly, the displacements along the other directions are also computed and the ranges in other directions are observed. The maximum positive displacement along the north direction comes to $0.8 \mathrm{~cm}$ and negative displacement is $7 \mathrm{~cm}$, thus the maximum range of a day for a month comes to $7.8 \mathrm{~cm}$ (figure 8).

The maximum positive displacement along the east direction comes to $7 \mathrm{~cm}$ and negative displacement is also $7 \mathrm{~cm}$. Thus the maximum range of the day for the month along east direction is $14 \mathrm{~cm}$ (figure 9). 


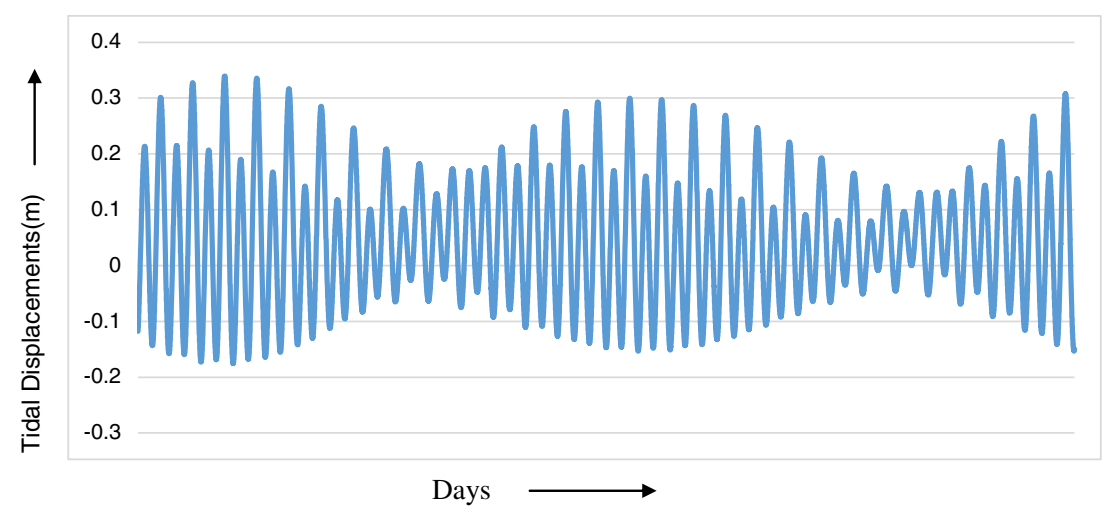

Figure 1. Tidal displacement along vertical direction at IGS Hyderabad.

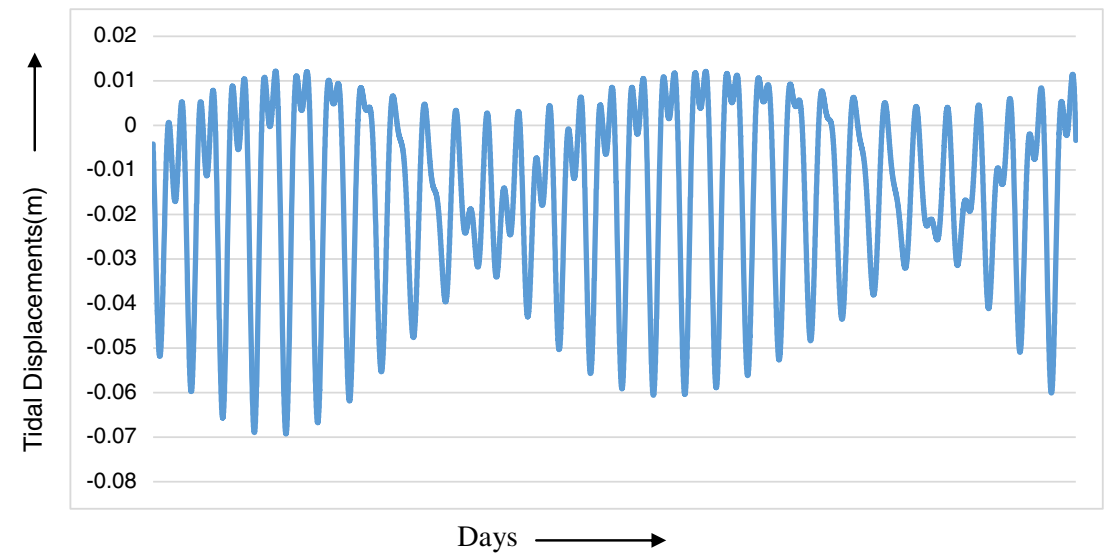

Figure 2. Tidal displacement along north direction at IGS Hyderabad.

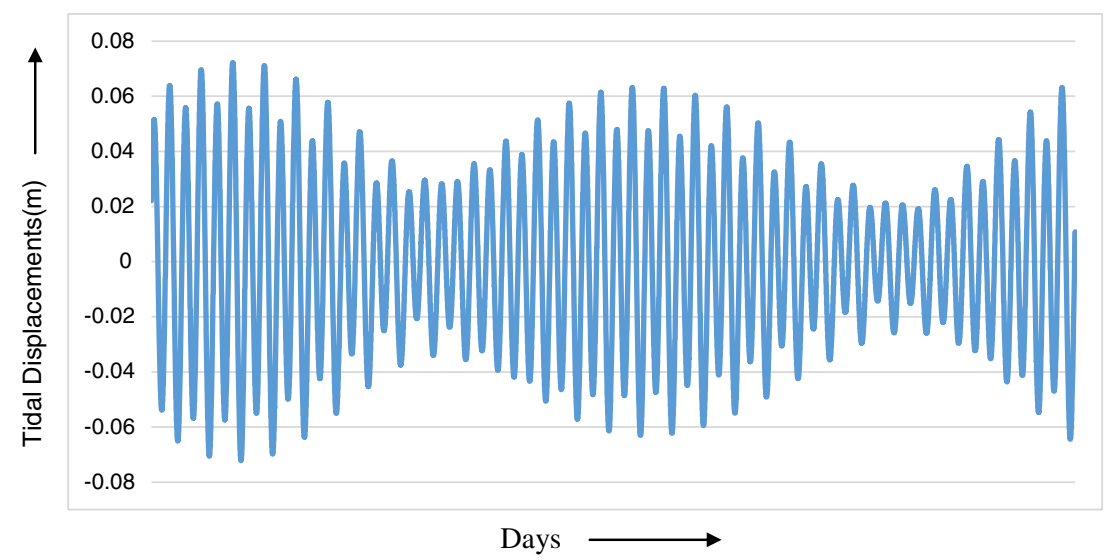

Figure 3. Tidal displacement along east direction at IGS Hyderabad.

\subsubsection{Comparative study between IGS stations at 1-min interval for a day}

The tidal displacements along all the three directions for a month are computed and shown individually for all IGS stations. Now to study the effect for a day, the displacements are computed for a day at IGS stations along all three directions as shown in figures 10, 11 and 12. This shows that how the earth surface is experiencing the effect and how tides are propagating from one station to another and with what speed. Tidal displacement is computed for 1 November, 2013 along all the three directions of all IGS stations.

It is clearly seen in figure 10 that along the vertical direction first the high tide reaches at IGS Beijing Fangshan station then IGS Hyderabad station and at last it reaches at IGS Ankara station. 


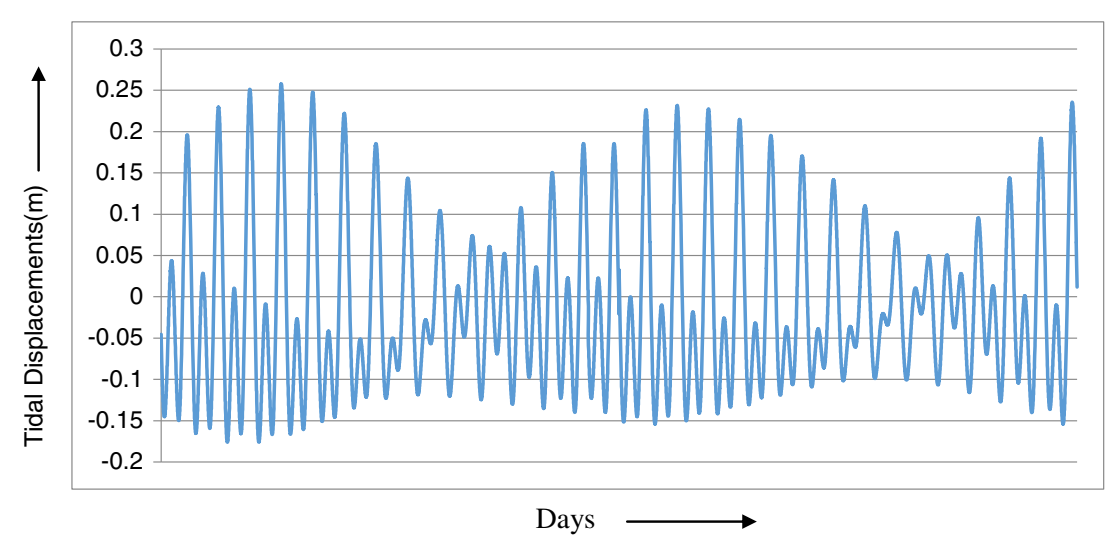

Figure 4. Tidal displacement along vertical direction at IGS Ankara.

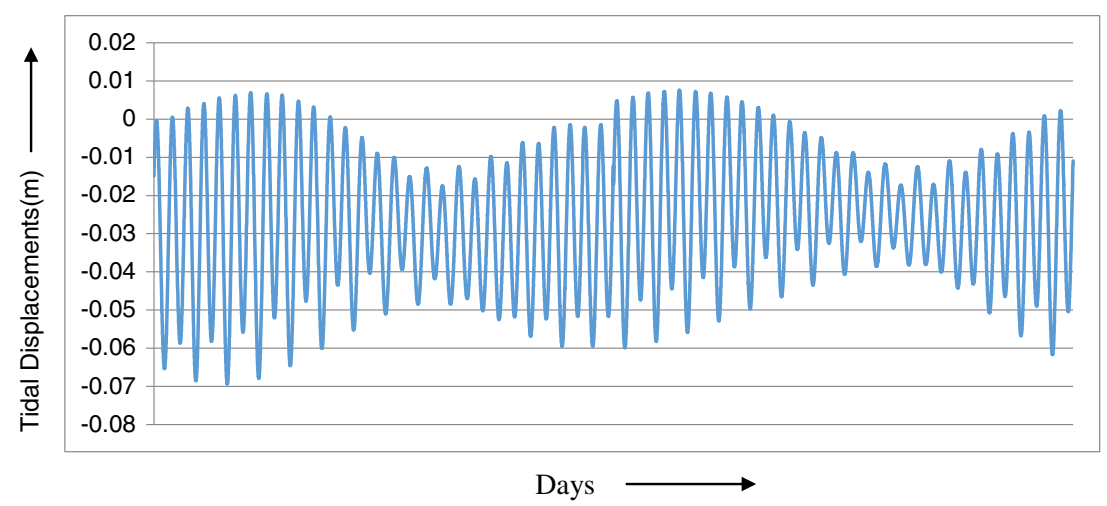

Figure 5. Tidal displacement along north direction at IGS Ankara.

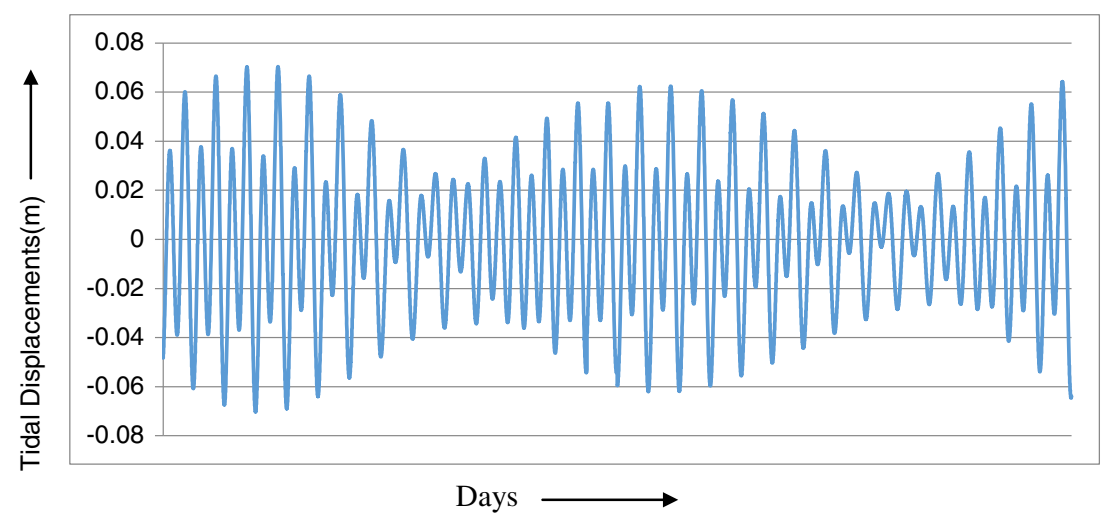

Figure 6. Tidal displacement along east direction at IGS Ankara.

The time lag between the stations is calculated. It comes to $187 \mathrm{~min}$ between IGS Hyderabad and IGS Ankara stations, 342 min between IGS Ankara and IGS Beijing Fangshan stations and $155 \mathrm{~min}$ between IGS Hyderabad and IGS Beijing Fangshan stations. We can easily compute the baseline distance between the stations through baseline processing. By this we can find out/calculate the propagation speed and direction of the solid earth tides on the surface of Earth.
Similarly, along the north direction first the low tide reaches IGS Beijing Fangshan station then IGS Hyderabad station and reaches the IGS Ankara station last (figure 11). The time lag of low tides between IGS Hyderabad and IGS Ankara comes to 187 min, between IGS Ankara and IGS Beijing Fangshan is 342 min and between IGS Hyderabad and IGS Beijing Fangshan is 155 min. Through these values we can easily compute the propagation velocity of tides on solid earth. 


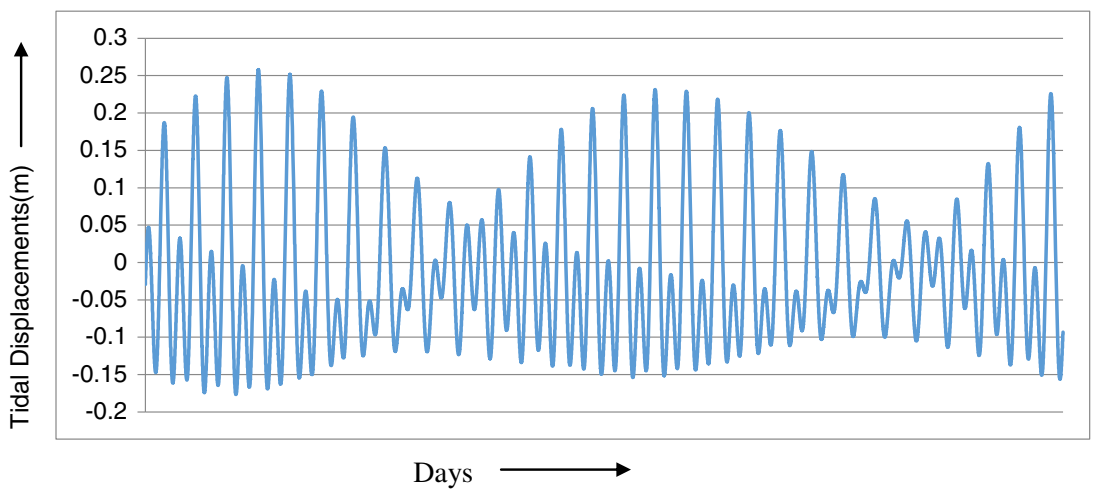

Figure 7. Tidal displacement along vertical direction at IGS Beijing Fangshan.

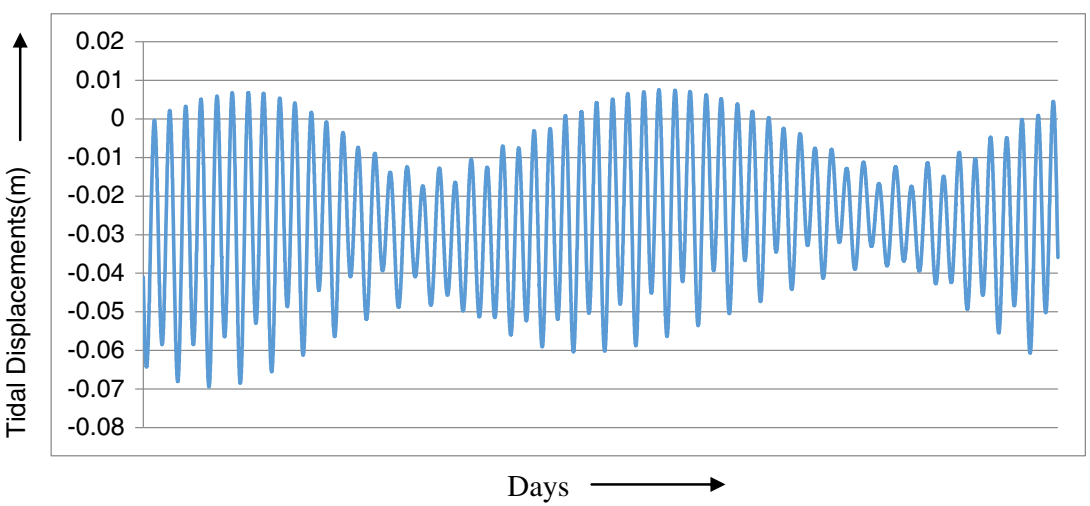

Figure 8. Tidal displacement along north direction at IGS Beijing Fangshan.

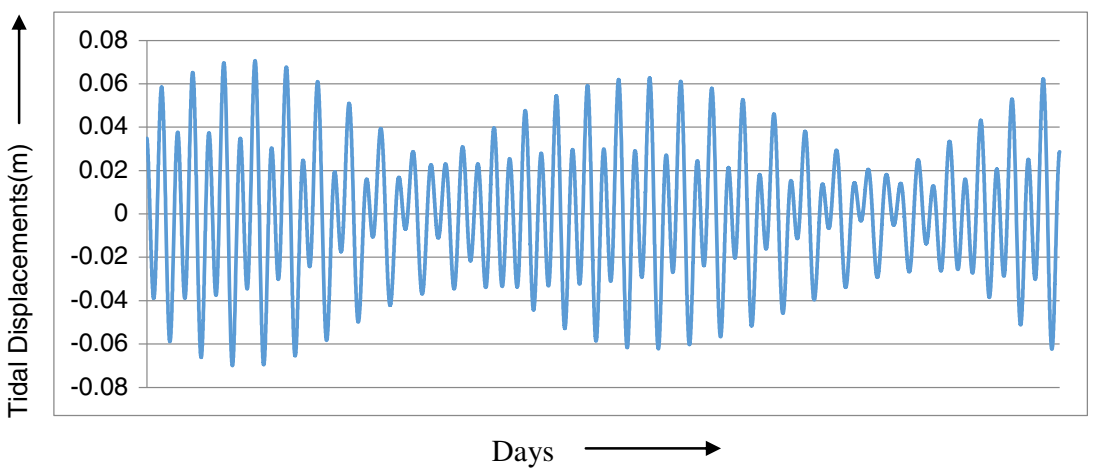

Figure 9. Tidal displacement along east direction at IGS Beijing Fangshan.

Similarly, along the east direction first the low tide reaches IGS Beijing Fangshan station then IGS Hyderabad station and reaches the IGS Ankara station last (figure 12). The time lag of high tides along east direction between IGS stations is the same as in the case of vertical and north directions.

\section{Discussion and results}

The study shows the variation in the position of the IGS station for the month of November. The results shown by the program 'Solid' indicate the displacements of the station for the particular day. For IGS Hyderabad station, the range of the station for a particular day along the vertical direction is about $48 \mathrm{~cm}$ with the maximum vertical displacement about $30 \mathrm{~cm}$ and minimum vertical displacement is about $-18 \mathrm{~cm}$. This maximum range occurs in approximately 6 hours. Along the north direction the displacement is $8 \mathrm{~cm}$, maximum displacement is $1 \mathrm{~cm}$, and minimum displacement is $-7 \mathrm{~cm}$. The displacement along the east direction is $14 \mathrm{~cm}$, with maximum $7 \mathrm{~cm}$ and minimum $-7 \mathrm{~cm}$. Similarly for IGS Ankara station, the maximum range displacement along vertical direction 


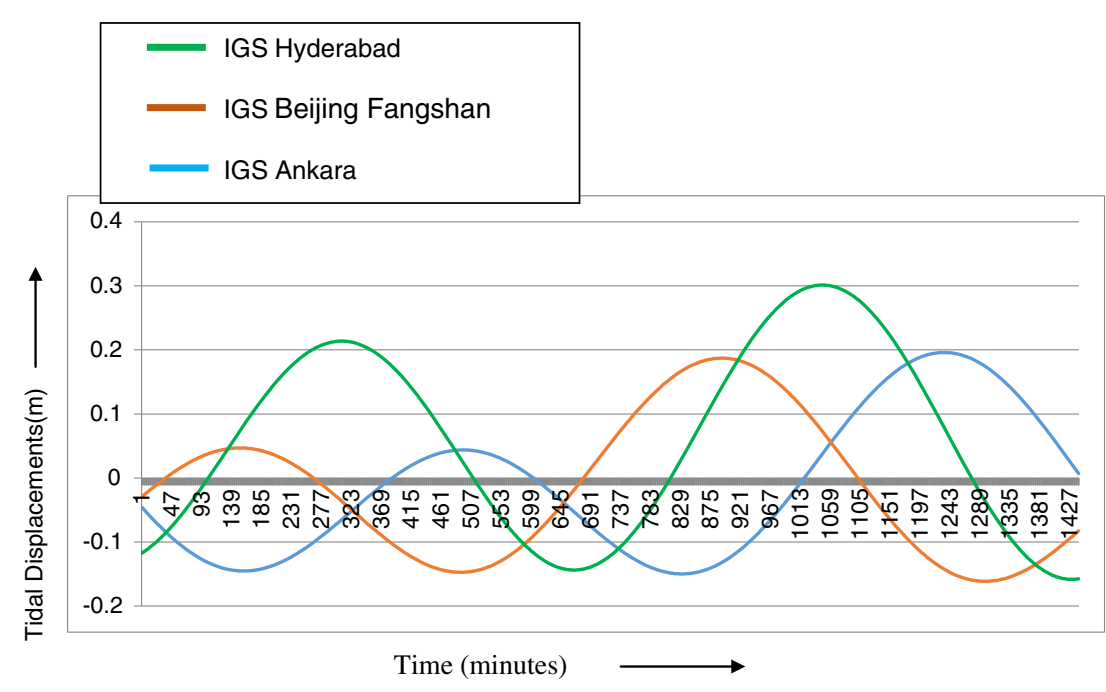

Figure 10. Tidal displacements of IGS stations along vertical direction in a day.

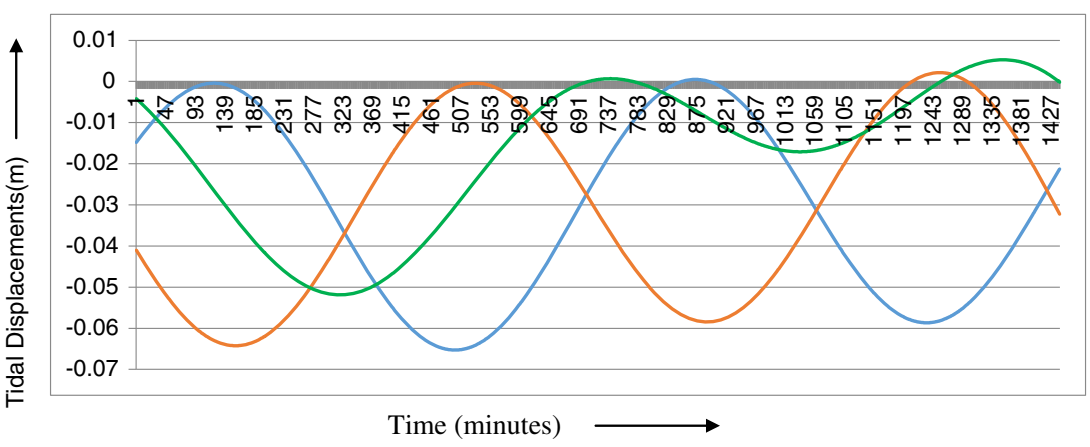

Figure 11. Tidal displacements of IGS stations along north direction in a day.

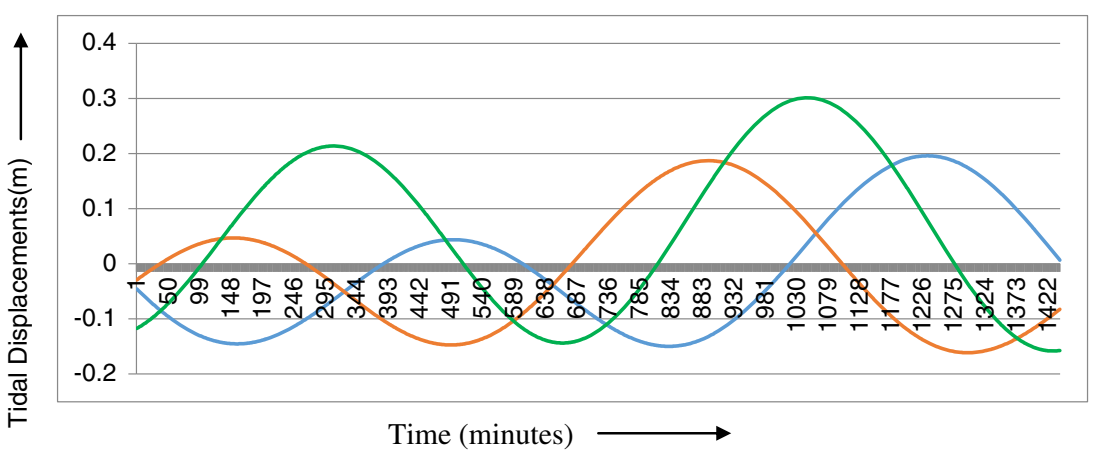

Figure 12. Tidal displacements of IGS stations along east direction in a day.

per day for a month comes to $43 \mathrm{~cm}$ with positive $23 \mathrm{~cm}$ and negative $17 \mathrm{~cm}$. Along north direction, the range comes to $7.7 \mathrm{~cm}$ with positive $0.7 \mathrm{~cm}$ and negative $7 \mathrm{~cm}$. Along east direction, the range comes to $14 \mathrm{~cm}$ with positive $7 \mathrm{~cm}$ and negative 7 $\mathrm{cm}$. Similarly, for IGS Beijing Fangshan, the maximum range displacement along vertical direction per day for a month comes to $42 \mathrm{~cm}$ with positive $25 \mathrm{~cm}$ and negative $17 \mathrm{~cm}$. Along north direction, the range comes to $7.8 \mathrm{~cm}$ with positive $0.8 \mathrm{~cm}$ and negative $7 \mathrm{~cm}$. Along east direction, the range comes to $14 \mathrm{~cm}$ with positive $7 \mathrm{~cm}$ and negative $7 \mathrm{~cm}$. Thus the position of the particular point is being affected continuously due to the solid earth tide. The variation at $1 \mathrm{~min}$ interval is calculated and it shows that there is a large variation in the position of the point due to the solid earth tides.

The one day comparison is done between IGS stations at $1 \mathrm{~min}$ intervals along all the three directions to observe the difference in time between 


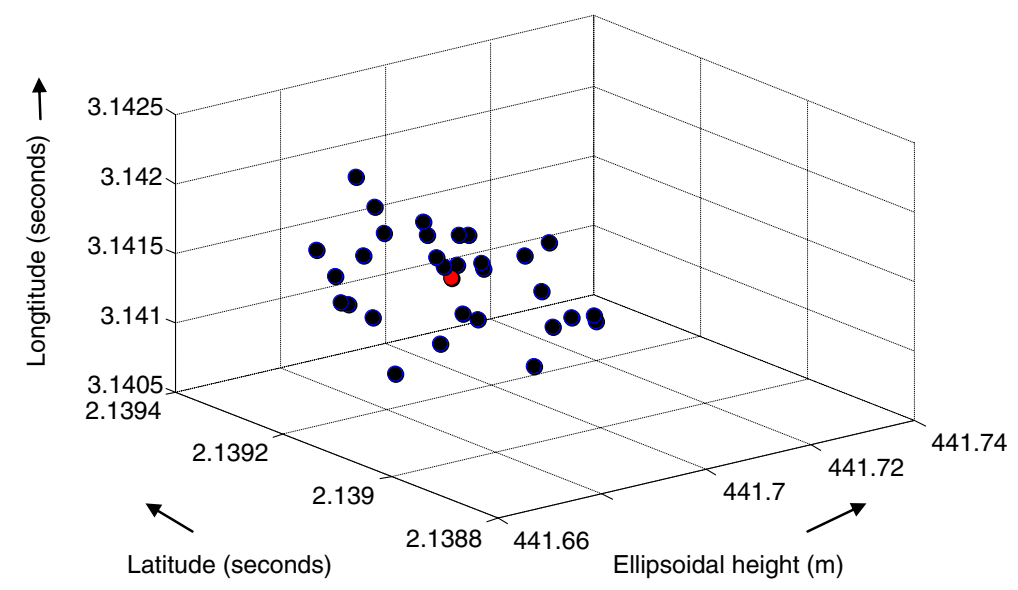

Figure 13. Positional variation of the coordinates due to solid earth tides where $\bullet$ indicates the positions (30 days) of the station for the month of November and o indicate the mean value of the coordinates for the month of November at IGS Hyderabad.

high tides of the IGS stations. The time lag between IGS Hyderabad and IGS Ankara stations comes to $187 \mathrm{~min}$, between IGS Ankara and IGS Beijing Fangshan is $342 \mathrm{~min}$ and between IGS Hyderabad and IGS Beijing Fangshan station is $155 \mathrm{~min}$. From this knowledge and by doing the baseline processing between these stations the propagation velocity of tides on solid earth can easily be computed.

But to study the effect of these continuous displacements on the coordinates of the point on daily basis, the static Precise Point Positioning (PPP) method is used. This method is implemented by GPS software Bernese 5.0. This software gives very accurate GPS coordinates and gives only a single mean value of the coordinates for a particular day (figure 13).

\subsection{By Precise Point Positioning (PPP)}

The GPS data of IGS Hyderabad is taken for the month of November 2013. It is processed by Bernese 5.0 and the coordinates are calculated daily. The graphic representation of the coordinates shows that how the regular displacements of the point are affecting the coordinates of the station on daily basis (figure 13).

The variation in the vertical ellipsoidal height within a month is discussed by the graphic representation. The variation in height (meter) is due to the regular displacements occuring due to solid earth tides. The value of geodetic undulation $(\mathrm{N})$ varies regularly but with very less quantity. Thus for all practical purposes, the value is assumed to be constant. Therefore the variation in the geodetic height and ellipsoidal height will be same. The data shows that 3rd November has the lowest height. The new moon (spring tide) is also on November
3. It means that due to the spring tide the tidal force is maximum, due to the maximum pull, the Earth's surface gets compressed due to which the height gets reduced to the lowest. Rotation period of the Moon around Earth is approximately 24 days. Therefore in approx. 12 days each maximum high and low tide will occur. After 3rd November, the next high tide is around 14 November. Same is observed for the 9th and 21st November. The range of the displacement along the vertical height comes to $3.52 \mathrm{~cm}$ for the month of November.

The latitude and longitude for any station is calculated in degree minutes and seconds. For studying the variation along the north and east direction the seconds value is taken. The range in the variation of the latitude, i.e., along the north side is very less. The variation of the latitude for the month is 0.0004 seconds. It is about $1.23 \mathrm{~cm}$. For one degree change in latitude near the equator the distance is about $110.567 \mathrm{~km}$. Therefore, the displacement along the north direction is about $1.23 \mathrm{~cm}$.

The variation in the magnitude along the east side is also very less. The range in the variation of the longitude for the month is 0.0009 seconds. This is about $2.73 \mathrm{~cm}$. For one degree change in longitude near the equator the distance is about $111.321 \mathrm{~km}$. Therefore, the displacement along the east direction for the month is about $2.73 \mathrm{~cm}$. The minimum value of the longitude on November 3 is also because of the spring tide.

The positional variation of the IGS station for the month of November is shown in figure 13. This figure shows that how the position of the station is varying continuously due to the solid earth tides.

This figure shows that how the position of the station varies continuously due to the solid earth tides. Therefore there is a high need to know the 
exact behaviour of the solid earth tides so that the corrections can be applied to the GPS readings for finding the exact value of the GPS coordinates for a specified place and time.

\section{Conclusion}

The Earth station displacement caused by the solid body tides, is the correction which must be observed for determining the Earth station's position. Tidal oscillation has a strictly definite periodicity, therefore, the desired corrections can be applied for any instant of time. The study of the earth tides helps us in understanding the response of the Earth's body to the internal and external forces.

\section{References}

Agnew D C 2007 Earth Tides; University of California, San Diego, CA, USA.

Dehant V, Defraigne P and Wahr J M 1999 Tides for a convective Earth; J. Geophys. Res. 104 1035-1058.

Farrell W E 1972 Deformation of the earth by surface loads; Rev. Geophys. Space Phys. 10 761-797.

Fu G and Sun W 2007 Effects of lateral in homogeneity in a spherical Earth on gravity earth tides; J. Geophys. Res. 112 B06409, doi: 10.1029/2006JB004512.

Haritonova Diana 2012 Solid Earth Tides in the Territory of Lativa; Riga Technical University.

Lambert A, Pagiatakis S D, Billyard A P and Dragert H 1998 Improved ocean tide loading corrections for gravity and displacement: Canada and northern United States; J. Geophys. Res. 103(30) 231-244.

Lambeck K 1988 Geophysical Geodesy: The Slow Deformations of the Earth; Oxford Science Publications, 718p.

Love A E H 1944 Treatise on the Mathematical Theory of Elasticity; 4th edn, Cambridge University Press.

Longman I M 1963 A Green's function for determining the deformation of the earth under surface mass loads: 2 . Computations and numerical results; J. Geophys. Res. 68 485-495.

Mathews P M, Dehant V and Gipson J M 1997 Tidal station displacements; J. Geophys. Res. 102(B9) 20,469-20,478.

Mantovani M S M, Shukowsky W, Freitas S R C and Neves B B B 2005 Lithosphere mechanical behavior inferred from tidal gravity anomalies: A comparison of Africa and South America; Earth Planet. Sci. Lett. 230 397-412.

McCarthy D and Petit G (eds) 2004 IERS Conventions (2003); IERS Technical Note No. 32, Verlagdes Bundesamts fur Kartographie und Geodasie, Frankfurt am Main, 127p.

Milbert Dennis 2011 Solid Earth tide; http://home.comcast. net/ dmilbert/softs/solid.htm.

Munk W H and Cartwright D E 1966 Tidal spectroscopy and prediction; Phil. Trans. Roy. Soc. London, Ser. A $259533-581$.

Munk W H and MacDonald G J 1975 The Rotation of the Earth: A Geophysical Discussion; 1st edn, Cambridge University Press, Cambridge, 323p.

Melchoir P 1983 The Tides of the Planet Earth; 2nd edn, Pergamon Press, Oxford, 641p.

Penna N T and Stewart M P 2003 Aliased tidal signatures in continuous GPS height time series; Geophys. Res. Lett. 30(23) 2184, doi: 10.1029/2003GL018828.

Penna et al. 2015 GPS height time series: Short period origins of spurious long period signals; J. Geophys. Res., under Review.

Richter B and Warburton R 1998 A new generation of superconducting gravimeters enables the in situ detection and elimination of offsets and interruptions from continuous gravity measurements; In: Proc. 13th Int. Symp. Earth Tides Brussels (ed.) Ducarme B, pp. 545-555.

Saito M 1967 Excitation of free oscillations and surface waves by a point source in a vertically heterogeneous Earth; Earth. J. Geophys. Res. 72 3689-3699.

Stewart M P, Penna N T and Lichti D D 2005 Investigating the propagation mechanism of unmodelled systematic errors on coordinate time series estimated using least squares; J. Geodesy 79(8) 479-489, doi: 10.1007/s00190-005-0478-6.

Schenewerk M S, Marshall J and Dillinger W 2001 Vertical ocean-loading deformations derived from a global GPS network; J. Geodyn. Soc. Japan 47(1) 237242.

Wahr J M 1981 Body tides on an elliptical, rotating, elastic and oceanless Earth; Geophys. J. Roy. Astron. Soc. 64 677-703.

Yuan Linguo and Chao Benjamin F 2012 Analysis of tidal signals in surface displacement measured by a dense continuous GPS array; Earth Planet. Sci. Lett. 355-356 255, doi: 10.1016/j.epsl.2012.08.035.

Yuan L G, Ding X L, Zhong P, Chen W and Huang D F 2009 Estimates of ocean tide loading displacements and its impact on position time series in Hong Kong using a dense continuous GPS network; J. Geodesy 83(11) 9991015 . 